\title{
The Plasmid Mobilome of the Model Plant-Symbiont Sinorhizobium meliloti: Coming up With New Questions and Answers
}

\author{
ANTONIO LAGARES, ${ }^{1}$ JUAN SANJUÁN, ${ }^{2}$ and MARIANO PISTORIO ${ }^{1}$ \\ ${ }^{1}$ IBBM, Instituto de Biotecnología y Biología Molecular, CONICET - Departamento de Ciencias Biológicas, \\ Facultad de Ciencias Exactas, Universidad Nacional de La Plata, (1900) La Plata, Argentina; \\ ${ }^{2}$ Departamento de Microbiología del Suelo y Sistemas Simbióticos. Estación Experimental del Zaidín,
} Consejo Superior de Investigaciones Científicas (CSIC), Granada, Spain

ABSTRACT Rhizobia are Gram-negative Alpha- and Betaproteobacteria living in the underground that have the ability to associate with legumes for the establishment of nitrogen-fixing symbioses. Sinorhizobium meliloti in particular-the symbiont of Medicago, Melilotus, and Trigonella spp.-has for the past decades served as a model organism for investigating, at the molecular level, the biology, biochemistry, and genetics of a free-living and symbiotic soil bacterium of agricultural relevance. To date, the genomes of seven different S. meliloti strains have been fully sequenced and annotated, and several other draft genomic sequences are also available (http://www.ncbi.nlm.nih.gov/genome/genomes/1004).

The vast amount of plasmid DNA that $S$. meliloti frequently bears (up to $45 \%$ of its total genome), the conjugative ability of some of those plasmids, and the extent of the plasmid diversity has provided researchers with an extraordinary system to investigate functional and structural plasmid molecular biology within the evolutionary context surrounding a plant-associated model bacterium. Current evidence indicates that the plasmid mobilome in S. meliloti is composed of replicons varying greatly in size and having diverse conjugative systems and properties along with different evolutionary stabilities and biological roles. While plasmids carrying symbiotic functions (pSyms) are known to have high structural stability (approaching that of chromosomes), the remaining plasmid mobilome (referred to as the non-pSym, functionally cryptic, or accessory compartment) has been shown to possess remarkable diversity and to be highly active in conjugation. In light of the modern genomic and current biochemical data on the plasmids of S. meliloti, the current article revises their main structural components, their transfer and regulatory mechanisms, and their potential as vehicles in shaping the evolution of the rhizobial genome.

\section{EXTRACHROMOSOMAL REPLICONS IN S. MELILOTI: PLASMID CONTENT AND DIVERSITY}

Bacteria grouped within the Rhizobiaceae, Phyllobacteriaceae, and Bradyrhizobiaceae families, collectively known as rhizobia, inhabit the soil under free-living conditions and are associated in symbiosis with the root of legumes as nitrogen-fixing organisms. Rhizobia do not form a single taxonomic cluster. Instead, they are distributed within distantly related lineages within the

Received: 21 April 2014, Accepted: 23 May 2014,

Published: •....•

Editors: Marcelo E. Tolmasky, California State University, Fullerton, CA, and Juan Carlos Alonso, Centro Nacional de Biotecnología, Cantoblanco, Madrid, Spain

Citation: Lagares A, Sanjuán J, Pistorio M. 2014. The plasmid mobilome of the model plant-symbiont Sinorhizobium meliloti: coming up with new questions and answers. Microbiol Spectrum 2(4):PLAS-0005-2013. doi:10.1128/microbiolspec.PLAS-0005 -2013 .

Correspondence: Antonio Lagares, lagares@biol.unlp.edu.ar (c) 2014 American Society for Microbiology. All rights reserved. 
alpha- and beta-subdivisions of the proteobacteria. The superb nitrogen-fixing capacity of the rhizobia-legume symbioses argues for the use of rhizobia for the introduction of nitrogen into agricultural soils, as a means of avoiding the massive use of chemical fertilizers $(\underline{1}, \underline{2})$.

A common feature of the rhizobial genome is that, in addition to the chromosome, plasmids are usually present that carry genetic material encoding widely diverse functions $(\underline{3}, \underline{4})$. The plasmids carrying genes involved in the symbiotic process have been named symbiotic plasmids or pSyms $(\underline{5}, \underline{6}, \underline{7})$. In addition to these symbiotic elements, rhizobia may carry other plasmidsreferred to as nonsymbiotic plasmids, non-pSyms or the functionally cryptic or accessory compartment-that are not indispensable for symbiosis or have simply not yet been assigned a specific function. That any of these plasmids may be lost, recovered, or also change their copy number or the contents of their genetic information is consistent with the plasmid's role as transient vectors of genomic structural change(s) within a given bacterial population ( $\underline{8})$.

In many rhizobia, plasmid DNA could constitute a substantially high percentage of the bacterial DNA, as in Sinorhizobium meliloti where the contribution of (mega)plasmid DNA is ca. $45 \%$ of the total. S. meliloti is a model species for studying plant-bacteria interactions and, in particular, rhizobia-legume symbiosis and symbiotic nitrogen fixation. In the type strain $S$. meliloti 1021 the genome consists of one chromosome of 3.65 $\mathrm{Mbp}$ and two symbiotic megaplasmids, pSymA and pSymB, that are 1.36 and $1.68 \mathrm{Mbp}$, respectively. In many other strains non-pSym (i.e., cryptic) plasmids of different sizes have also frequently been reported $(\underline{9}, \underline{10}$, $11,12,13,14$ ) (also, see further on).

\section{SYMBIOTIC (MEGA) PLASMIDS (pSyms)}

As mentioned above, pSyms are those plasmids that bear essential genes for the establishment of a fully functional symbiotic nodule in the legume host. The complete genetic sequencing of the model strain S. meliloti 1021 more than 10 years ago enabled a knowledge of the gene content of both these $\mathrm{pSym}$ plasmids $(\underline{15}, \underline{16})$. The availability of such information furthered progress in the development of new tools for the functional study of symbiosis, which, in turn, shed additional light on the structure and modular conformation of both megaplasmids. This novel information deepened our understanding of how a symbiosis such as this model association represents could have evolved $(\underline{15}, 16)$. pSymA codifies genes required for nodulation (nod), for the nitrogenase (nif), and for nitrogen fixation ( $f i x[15])$. In addition, pSymA carries genes likely to be involved in nitrogen and carbon metabolism, transport, and stress and resistance responses that give $S$. meliloti an advantage in its specialized niches $(\underline{15})$. The determination of the S. meliloti 1021 complete genomic sequence indicated that pSymA could have been acquired by horizontal gene transfer (HGT) because of the megaplasmid's lower GC content $(60.4 \%)$ and its different codon usage compared with the other replicons (i.e., the bacterial chromosome and pSymB).

pSymB was found to bear genes encoding soluteuptake systems along with the enzymes involved in both polysaccharide biosynthesis and catabolic activities. pSymB also exhibited many features of a typical chromosome such as the presence of essential housekeeping genes including those encoding the arginine tRNA, minCDE cell division, and the asparagine-synthetic pathway. In contrast, no essential gene could be mapped on pSymA.

Giuntini et al. (17) applied comparative genomic hybridization on an oligonucleotide microarray in order to estimate genetic variations in four natural strains of $S$. meliloti compared with the type strain 1021 . For the analysis, they used two strains obtained from Italian agricultural soils and two isolated from a desert soil in the Aral Sea region. The assay showed that the largest amount of genetic polymorphism-i.e., the genes that were found to be more variable in the comparison between the type strain 1021 and the field isolates- was present in pSymA (17). Gene diversity was not randomly distributed among the three replicons (pSymA, pSymB, and chromosome) in the four natural isolates. Consistent with this, pSymA showed a higher density of insertion sequence elements and phage sequences compared with pSymB (15). In general, transposable elements tend to accumulate in regions where they do not disrupt essential genes. All these data suggest that $\mathrm{pSymA}$ is a plasmid-like replicon, whereas $\mathrm{pSymB}$ has several chromosomal characteristics. This conclusion was also supported by the observation that pSymA could be cured in S. meliloti 2011, whereas pSymB could not (18). The pSymA-cured derivative strain showed no difference in its growth behavior compared with the wild-type strain in both complex and defined media, but otherwise was unable to use a number of substrates as a sole source of carbon on defined media (18). Slater et al. (19) proposed a common mechanism of secondary chromosome formation in Rhizobiaceae and other bacteria. A prerequisite for this evolution was proposed to be the intracellular presence of a second replicon capable of stably and efficiently replicating large DNA molecules. 
Current items of evidence taken together support the notion of a near chromosome status for pSymB (i.e., one of a so-called chromid [20, 21]). The genes present in $\mathrm{pSymB}$ were recently observed to be more widespread in different taxa than those encoded by the other $S$. meliloti replicons (20). Within such a context, pSymB was proposed to have a role in intraspecies differentiation through positive gene selection, whereas pSymA was suggested to contribute to the emergence of new functions (20). Another recent investigation compared the full genomic sequence of three strains of $S$. meliloti: 1021, AK83, and BL225C (22). The pSymB showed a high resistance to genomic rearrangements, demonstrating an almost perfect shared synteny. Only strain AK83 presented few rearrangements in certain regions of its pSymB. Within pSymA, however, a very low degree of synteny was observed, thus indicating a much greater rate of rearrangements. When the S. meliloti SM11 genome (23) was included in the analysis, plasmid pSmeSM11c (the equivalent to the pSymA of strain 1021) presented the most diverse information in comparison with the pSymA homologs from strains AK83 and BL225C (22). Another interesting feature of pSmeSM11c was that large gene regions proved to be closely related to regions in different plasmids from strain Sinorhizobium medicae WSM419. In addition, pSmeSM11c carries other novel gene regions, such as additional plasmid-survival genes; the acdS gene involved in modulating the level of the phytohormone ethylene; and a set of genes with predicted functions associated with degradative capabilities, stress response, and amino-acid metabolism and associated pathways (23).

The higher structural variation in $\mathrm{pSymA}$ is also consistent with its potential self-transmissibility via conjugation (24), compared with the pSymB, whose mobilization depends on the type IV secretion system present in the pSymA megaplasmid (25). Later on in this review, we will discuss the regulatory aspects associated with the conjugative mobilization of both of these pSyms.

\section{NON-pSym (CRYPTIC OR ACCESSORY) PLASMID REPICONS IN S. MELILOTI}

The size of the non-pSym plasmids is highly variable, ranging from ca. $7 \mathrm{~kb}(\underline{26})$ to up to $260 \mathrm{~kb}(\underline{22})$. Certain regions of these plasmids may be involved in the emergence of genetic rearrangements $(\underline{27}, \underline{28})$ owing to the presence of insertion sequences, the generation of deletions, or plasmid cointegrations. The information available, mainly stemming from sequence data, indicates that the non-pSyms may encode phenotypic features providing the rhizobia with adaptive advantages. NonpSym plasmids are thought to encode functions that most likely impact the environmental fitness of the rhizobia. For this reason, the observation that the phenotypic changes resulting from gene knockouts in these plasmids proved to be difficult to study under laboratory conditions was certainly logical. Stiens et al. (11) isolated the plasmid pSmeSM11a from a dominant indigenous $S$. meliloti subpopulation within the context of a longterm field-release experiment involving genetically modified S. meliloti strains in Germany. An analysis of the plasmid sequence revealed that approximately two-thirds of pSmeSM11a was occupied by accessory genetic modules that could be providing adaptive advantages or broadening the host bacterium's responsive spectrum. Therefore, the authors postulated that the presence of pSmeSM11a might have been responsible for the dominance of strain SM11. The finding that non-pSym plasmids may encode information for the metabolism of soil compounds or root exudates suggested that these plasmids could play a significant role in the free life cycle of the rhizobia and generated speculation on future possibilities for using the plasmid information for enhancing rhizobial capabilities to colonize rhizospheric environments. In addition, reports have indicated that the presence of non-pSym plasmids may be related to nonessential symbiotic capacities. For example, Bromfield et al. (29) found that the presence of the cryptic plasmid pTA2 stimulated competitiveness for nodulation, and genes directly involved in nodulation efficiency have been described on the plasmid pRmeGR4b of $S$. meliloti GR4 $(\underline{30}, 31, \underline{32})$. By contrast, Velázquez et al. (33) reported that the development of fully effective root nodules in alfalfa by $S$. meliloti strain SAF22 was attenuated by the presence of the cryptic plasmid pRmSAF22c, which was interfering with the normal nodular development necessary to sustain a fully effective nitrogen-fixing symbiosis. Thus, an understanding of how these rhizobia become adapted to changing environments (soil and plant host) will clearly require a more solid characterization of the non-pSym mobilome, because those plasmids appear to be a main cellular trans source of new functions.

\section{THE DIVERSITY OF NON-pSym PLASMIDS IN S. MELILOTI}

Over the years, different investigations were aimed at examining the genomic diversity of bacteria associated with the genus Medicago, paying special attention to the presence of cryptic plasmids in the associated rhizobial 
strains. The most convenient technique for examining large plasmids—such as those borne by $S$. meliloti-was developed by Eckhardt (34) and was based on the lysis of the bacterial cells within an agarose gel (lysis in situ). Since under those conditions of minimal mechanical stress (i.e., the shear forces applied) little physical damage to the plasmids occurs, they are released intact in the lysate before migration in the gel. Several modifications of the original technique have been described $(\underline{7}, \underline{35}, \underline{36})$ and have been used accordingly to screen for the presence of large plasmids in rhizobia.

Within the context of the genomic analysis of a collection of $S$. meliloti isolates from Germany by IS fingerprinting, Kosier et al. (12) demonstrated the existence of a high diversity of plasmid profiles in Eckhardt gels. In that study, $78 \mathrm{~S}$. meliloti field strains were first grouped into 18 different plasmid profiles with $96 \%$ of the isolates containing cryptic plasmids. The most common plasmid profile exhibited a ca. $250 \mathrm{~kb}$ band that was present in 18 strains (23\%). In another study aimed at investigating the symbiotic characteristics of $S$. meliloti populations present in soils with different agricultural histories, Velázquez et al. (37) isolated rhizobia from three different sites in Salamanca, Spain. In each soil, 7 plasmid profiles were found with 13 different plasmid profiles being observed overall. None of these earlier studies, however, characterized whether or not the plasmid profiles obtained were representative of the existing plasmid diversity within the populations investigated. Pistorio et al. (13) gathered a collection of $S$. meliloti isolates that, while remaining manageable and practical from an experimental point of view, contained a good representation of the plasmid diversity present in the soils sampled. Those authors used $S$. meliloti isolates recovered from 25 different soils of central Argentina to investigate whether any correlation existed between the overall genomic constitution of the rhizobia and the content of cryptic plasmids present in those same bacteria (see the following section). The analysis by Eckhard-like gels of the plasmid content of 64 isolates resulted in 22 different plasmid profiles with at least 38 discrete plasmid bands. In order to evaluate to what extent the local diversity of plasmid profiles was represented in the collection of those isolates, the cumulative Shannon-Weaver index (38) was calculated, and the resulting values for a given number of strains $(n>49)$ reached a plateau. The asymptotic behavior of the cumulative Shannon-Weaver index, for the region of higher $n$ values, indicated that the different plasmid profiles from the $S$. meliloti collection constituted a satisfactory representation of the existing plasmid diversity ( $\underline{13})$.

\section{REPLICATION SYSTEMS PRESENT IN THE S. MELILOTI pSym AND NON-pSym PLASMIDS}

The best studied replication genes in soil bacteria belong to the repABC system. More specifically, within the Rhizobiaceae family these repABC-type replicons predominate in the plasmids whose replication regions have been investigated $(39, \underline{30})$. The system is named according to the characteristic genetic arrangement of repA-repB-repC that, as such, conforms an operon (41). The RepA and RepB have predicted amino acid sequences similar to those of proteins related to plasmid partition; the repA genes encode ATPases of the ParA family, while the $r e p B$ genes encode for the $p a r S$-binding proteins ParB. The parS stretch is a centromere-like sequence and can be located at different positions within the rep $A B C$ operon. One general model suggests that the ParA (RepA) proteins polymerize into filaments, and ParB (RepB) binds both the parS region and ParA, thus acting as an adaptor between the plasmids and the filaments that are responsible for the segregation process (42). An alternative mechanism suggests a diffusionratchet model, where ParB associates with the plasmid chases and redistributes the ParA gradient on the nucleoid, which in turn mobilizes the plasmid (43). The RepC is essential for plasmid replication as a replicationinitiator protein $(39,41)$. The large intergenic sequence between $r e p B$ and rep $C$ contains a gene encoding a small antisense RNA (ctRNA). This RNA is a transincompatibility factor, modulating the RepC levels and thereby the resulting plasmid copy number $(44,45, \underline{46}$, 47). Current genomic data indicate that most $S$. meliloti plasmids described so far bear rep $A B C$ replication cassettes. Other plasmids bear a repC replication cassette without partition components and are evolutionarily related to the rep $A B C$ family, because they bear a RepC replication-initiator protein $(\underline{48}, \underline{49})$. In this family, an antisense RNA also plays a central role as a negative regulator of the expression of repC, and is a transincompatibility determinant as well (49).

Other plasmid-replication systems have been found to be present in S. meliloti. One includes only one member, the 7.2-kb plasmid $\mathrm{pRm} 1132 \mathrm{f}$ isolated from strain S. meliloti 1132. This replication system belongs to group III of the rolling-circle-replication plasmids (26). Finally, Watson and Heys (50) isolated a plasmid replication region from strain $S$. meliloti MB19 that contained genes similar to those associated with several broad-host-range plasmids. The Rep protein in this instance was related to the one present in the broad-hostrange plasmid pVS1 from Pseudomonas aeruginosa. 
CONJUGATIVE PROPERTIES OF THE NON-pSym PLASMID MOBILOME IN S. MELILOTI: THE FREQUENCY AT WHICH NON-pSym PLASMIDS ARE FOUND TO BE TRANSMISSIBLE-EVIDENCE IN SUPPORT OF A STRIKINGLY ACTIVE MOBILOME

HGT, homologous recombination, and gene conversion appear to be central mechanisms contributing to the shaping of genomic structures in rhizobia $(13,24,25$, $\underline{51}, \underline{52}, 53,54,55,56,57,58,59)$. As mentioned above, $S$. meliloti isolates frequently bear a varying and significant amount of non-pSym-plasmid DNA (12), which in several instances has been reported to be either mobilizable or self-transmissible via conjugation $(\underline{9}, \underline{14})$. In direct relevance to these considerations, a publication by Bailly et al. (60) aimed at investigating the diversity of bacteria associated with plants of the genus Medicago provided strong evidence in support of an active HGT involved in modeling the genomes of the symbionts $S$. meliloti and $S$. medicae. All examples of this type made the transferable character of the less studied nonpSym plasmids in S. meliloti an issue of central concern for investigation because of their possible role as vehicles of adaptation, evolution, and diversification. Thus, using a collection of $S$. meliloti isolates recovered from humic soils of Argentina, Pistorio et al. (13) studied the percentage of rhizobia that carried transmissible plasmids, the presence of helper functions, and the incidence of phenomena restricting plasmid entry. In that work, the quantitative estimation of diversity was numerically analyzed by means of the Shannon-Weaver (61) and the Simpson (62) indices through the use of different operational taxonomic units (OTUs) that were indicative of: (i) the kind of plasmid profiles present, (ii) the type of PCR fingerprints (i.e., genomic variants), and (iii) possible variants with respect to the combinations of plasmid profiles and genomic fingerprints within the collection of isolates. Interestingly, no clear evidence of association (linkage) was obtained between particular genomic backgrounds and their accompanying plasmids. This result thus argued for the existence of an active horizontal exchange of plasmids, the occurrence of active recombinational events, or both. In order to investigate how active the plasmid transfer within the collected diversity was, a systematic search for transmissible plasmids was performed by screening representative $S$. melilot $i$ isolates from each of the 22 different plasmid profiles (i.e., the plasmid OTUs) identified. Transmissible plasmids could be found in $14 \%$ of the isolates representing the different plasmid OTUs, indicating the presence of either self-transmissible or mobi- lizable plasmids (13). Of the isolates representing one plasmid OTU each, 29\% were demonstrated to be capable of mobilizing the model plasmid pSmeLPU88b (9) to a third bacterial strain, thus providing strong evidence for the ubiquitousness of compatible helper functions within the S. meliloti isolates (13). Remarkably, such conjugative system could also be found in a strain from a distant geographic origin as well, as demonstrated by the ability of the European $S$. meliloti GR4 to mobilize plasmid pSmeLPU88b (9). Strain GR4 bears the self-transmissible plasmid pSmeGR4a, and the mobilizable plasmid pSmeGR4b $(14, \underline{63})$. Figure 1 presents a summary of the different events observed when plasmid pSmeLPU88b was transferred to an $S$. meliloti final recipient through the use of representative isolates from the different plasmid-diversity groups as potential donors. Certain isolates $(14 \%)$, although refractory to receiving the plasmid pSmeLPU88b, accepted other broad-host-range plasmids. Such behavior could be the consequence of surface exclusion and/ or either plasmid-replication or recipient-strain DNArestriction system incompatibility. Irrespective of these observations, the results taken together argued for a strikingly high proportion of the $S$. meliloti genotypes $(36 \%)$ bearing either transmissible plasmids or helper functions (see Fig. 1). The available evidence indicates that the non-pSym compartment in S. meliloti is a highly active plasmid mobilome.

Contrasting with the information on the conjugative character of several $S$. meliloti plasmids, little research has been done to characterize transfer frequencies of pSym and non-pSym plasmids in soil samples and in the field. Studies for the S. meliloti cryptic plasmid pSmeLPU88b (64), and those reported by Kinkle and Schmidt for the symbiotic plasmid (pSym) pJB5I from a pea symbiont Rhizobium leguminosarum bv. viciae (65), indicate that rhizobial plasmids with detectable mobilization frequencies in the laboratory are transferred in nonsterile rhizospheric soil at rates between $10^{4}$ and $10^{6}$ events/recipient bacteria, irrespective of their symbiotic or nonsymbiotic character. The evaluation of plasmid transfer in nature has been considered long since a key issue to understand the relevance of the horizontal gene flow in the adaptive responses of the bacteria. Precise estimations of plasmid mobilization frequencies with analysis of the transient host bacteria (including nonrhizobia) will be required, both in soil and the rhizosphere, in support of any future attempt to develop predictive models of conjugative gene dispersion within the natural rhizobial communities. 


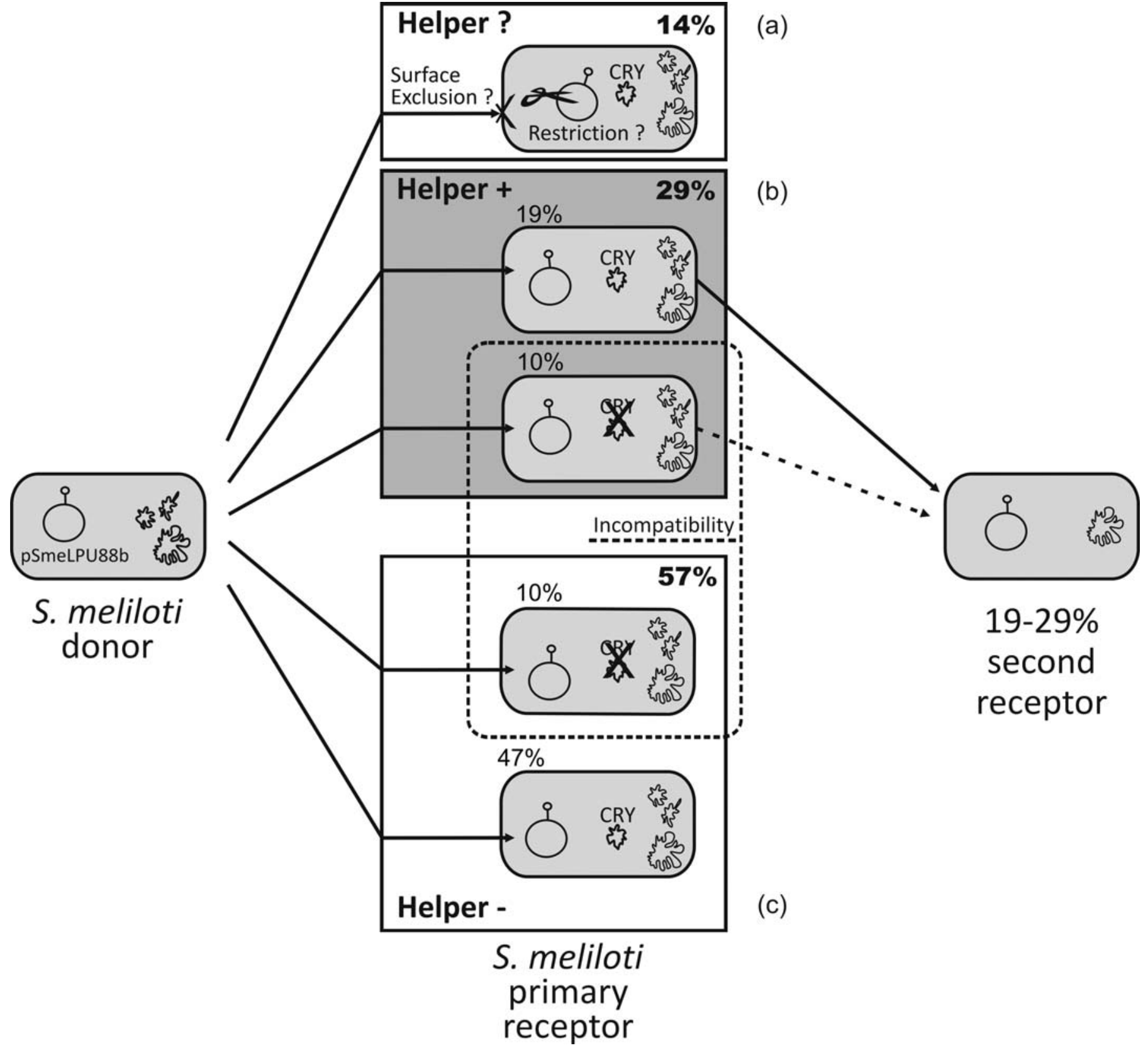

FIGURE 1 Scheme of the different possible destinies of the model plasmid pSmeLPU88b:: Tn5 when transferred to $S$. meliloti from different plasmid-diversity groups (plasmid OTU types; see reference 13 ). The percentage assigned to each event was calculated upon consideration of an abundance of plasmid OTU types in which all are equally represented. Box A represents those OTUs that proved unable to host plasmid pSmeLPU88b::Tn5 because of either surface-exclusion phenomena or plasmid restriction within the recipient cell. Box B represents OTUs bearing helper functions able to mobilize the model plasmid. Box $C$ represents isolates that, under our experimental conditions, were not able to mobilize plasmid pSmeLPU88b::Tn5. Some isolates belonging to the helper (+) class (Box B) or the helper (-) class (Box C) bear cryptic plasmids that display a replication incompatibility with plasmid pSmeLPU88b::Tn5 (dotted box). Cry, cryptic plasmid. The percent values on the right denote the maximal expected transfer frequency to a third bacterial strain (Reprinted from FEMS Microbiol Ecol [13] with permission of the publisher). doi:10.1128/microbiolspec.PLAS-0005-2013.f1 


\section{GENETICS OF CONJUGATIVE MODULES IN S. MELILOTI: pSym- AND NON-pSym-PLASMIDS}

Considerable efforts have been made to understand the genetics of the rhizobial conjugative systems (for a comprehensive summary, see the review by Ding and Hynes [66]). The available genomic information shows that the conjugative regions in rhizobia can be divided into four Dtr (DNA transfer and replication) types (I to IV) and three Mpf (Mating pair formation) types (I, II, and IV) according to their gene structure plus their associated regulatory mechanisms $(66,67,68)$. While the conjugative activities of the plasmids bearing type I Dtr/Mpf proved to be regulated by quorum sensing (e.g., plasmids pRetCFN42a from Rhizobium etli CFN42, pNGR234a from Sinorbizobium fredii NGR234, and pTiC58 from Agrobacterium tumefaciens C58), plasmids with type II Dtr/Mpf were found to have an RctArepressed conjugative regulation (e.g., the megaplasmid pSymA in strain S. meliloti 1021, and plasmids pRet $42 \mathrm{~d}$ and pAtC58). Finally, a strong association has been observed between each of the Dtr types (I through IV) and a specific kind of (cognate) relaxase, a correspondence that provides a strong indication of the coevolution of the genes within these conjugative elements in rhizobia. Figure 2 shows the phylogeny of representative rhizobial relaxases and how they correlate with each of the Dtr types.

In the specific case of $S$. meliloti, plasmids may bear a Dtr/Mpf belonging to any of the four types described in rhizobia (Fig. 2). The megaplasmids pSymA and pSymB from the model strain S. meliloti 1021 bear a type II Dtr/ $\mathrm{Mpf}$ (we will describe below how conjugation is regulated in these symbiotic megaplasmids). The pSymA homologs in strains S. meliloti SM11 and AK83 both carry, in addition to the type II, a type IV Dtr/Mpf first described for a non-pSym plasmid (67) and recently reclassified as type IVB (68). With respect to the non-pSym $S$. meliloti plasmids, most functional analyses have concentrated on the model plasmid systems $\mathrm{pSmeLPU} 88 \mathrm{a} / \mathrm{b}$ and $\mathrm{pSmeGR} 4 \mathrm{a} / \mathrm{b}$, both of which were also found to carry type IVB Dtrs/Mpfs (Fig. 2) $(67,69)$. The remarkable ubiquitousness of the Mpf functions able to mobilize pSmeLPU88b $(13, \underline{67})$ points to the S. meliloti type IVB Dtr as being a key component for gene exchange within the species. Interestingly, this type IVB Dtr has also been found in plasmids from other rhizospheric and soil bacteria that include Agrobacterium (e.g., pATS4a), Ochrobactrum (e.g., pOANT01), and Chelativorans (e.g., pBNC-01 [67]). In all these organisms, the type IVB Dtr presented a highly con- served synteny with a gene sequence (parA-like-oriTmobC-mobZ) (67). The oriT in plasmid pSmeLPU88b could be functionally mapped on a 278-bp fragment slightly overlapping with the mobC locus (67).

As occurs with the Dtrs I, II, and III, the Dtrs IVB also encode cognate relaxases whose phylogenies reveal a clearly distinct branch $\left(\mathrm{MOB}_{\mathrm{P} 0}\right)$, close to the $\mathrm{MOB}_{\mathrm{P} 3}$ and $\mathrm{MOB}_{\mathrm{P} 4}$ enzymes and separated from all other known $\mathrm{MOB}_{\mathrm{Q}}$-like rhizobial relaxases (67). Sequence analysis of the type IVB relaxase from plasmid pSmeLPU88b (designed MobZ) revealed the presence of three conserved motifs (I, II, and III) as in other relaxases (70). Within Motif I (amino acids 109 to 129) of the relaxase, as expected, a tyrosine (Y115) was present consistent with the requirement of this residue for the catalytic activity of the enzyme. Upon a profileprofile analysis and fold recognition, the residue Y115 aligned-within a predicted $\alpha$-helix-with the experimentally confirmed catalytic residue Y25 of the relaxase MobA from plasmid R1162 (67).

The bacteria carrying plasmids with relaxases of the $\mathrm{MOB}_{\mathrm{P} 0}$ group thus far reported inhabit soils, plants, or aquatic environments; with the sole exception of Ochrobactrum anthropi, which species (although also present in soil) has been found mainly in humans (71). The close phylogenetic relationship among the relaxases of the $\mathrm{MOB}_{\mathrm{P} 0}, \mathrm{MOB}_{\mathrm{P} 3}$, and $\mathrm{MOB}_{\mathrm{P} 4}$ clades suggests that their host plasmids might constitute frequent HGT vehicles among bacteria with overlapping environments (e.g., among soil- and/or plant-associated bacteria). At the moment, no evidence has been found for conjugative-transfer regulation in plasmids bearing the type IVB Dtrs/Mpfs. Recent evidence indicated that the conjugative transfer of plasmid pRleVF39b carrying a type IVA Dtr/Mpf is under repression control by a transcriptional-regulator protein (TrbR) whose sequence includes a helix-turn-helix xenobiotic-response element (66). Nevertheless, the existing genetic differences between types IVA and IVB Dtrs/Mpfs-plus the absence of a $\operatorname{tr} b R$ ortholog in the fully sequenced plasmid pSmeGR $4 a$ - both suggest that this regulation is likely not to be present in the $S$. meliloti plasmids bearing type IVB Dtrs/Mpfs.

Current genomic data on the S. meliloti extrachromosomal replicons revealed an increasing sequence variation from $\mathrm{pSymB}$ homologs $\rightarrow \mathrm{pSymA}$ homologs $\rightarrow$ non-pSym plasmids. Interestingly, the increasing genetic variation in each of these replicons parallels their likewise increasing conjugative-mobilization rates: from a strict conjugative control in the pSym plasmids to the lack of any evident conjugative regulation in some of the 


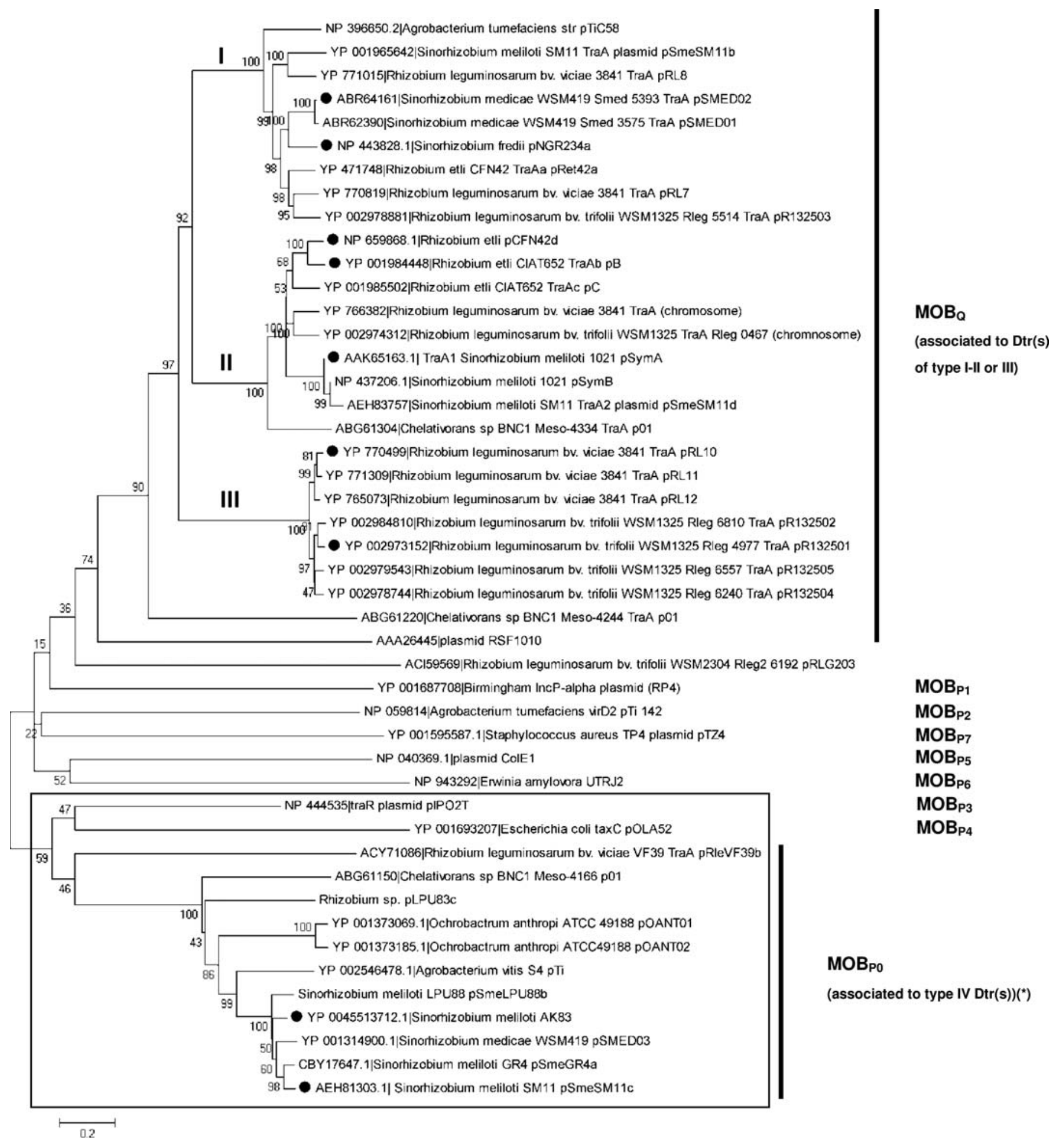


non-pSym S. meliloti plasmids (i.e., plasmids bearing type IVB Dtrs/Mpfs). Such a gradient of replicon plasticity strongly suggests that novel genes might likely reach rhizobia through the non-pSym mobilome, with only some of those genes being incorporated (after midand long-term selection) into structurally more stable replicons. An adaptive strategy of this type recalls the mosaic character of the rhizobial plasmid mobilome. A comparison of the nod-gene locations among the plasmids (see black dots in Fig. 2) with the phylogenetic topology of the rhizobial relaxases reveals that the symbiotic genes may be found on plasmids bearing any of the four Dtr types, thus indicating that pSym plasmids arise as independent chimeras not specifically associated with any particular mobilization system. A deeper sequencing of the $S$. meliloti plasmid mobilome will be necessary to better understand how gene exchange among replicons (and their further selection) operates over an evolutionary time scale.

\section{THE NEED FOR ADDITIONAL GENETIC DETERMINANTS OTHER THAN THE Dtr AND MPf REGIONS AS REVEALED BY THE STRAIN-DEPENDENT REQUIREMENT FOR MOBILIZATION OF PLASMID pSmeLPU88b IN S. MELILOTI}

The mobilization of pSmeLPU88b by plasmid pSmeLPU88a has recently been shown to require the newly identified gene rptA (for Rhizobium-plasmid transfer) in strain S. meliloti 2011, but not in strain LPU88 (72). A mutant carrying a disrupted copy of $r p t A$ in plasmid pSmeLPU88a was still able to facilitate the mobilization of plasmid pSmeLPU88b from strain S. meliloti LPU88, but not from strain 2011. Strikingly, no extra copies of the rptA gene were found by hybridization in strain LPU88. These observations raise new questions as to (i) what the alternative active components are that complement the deficiencies in $r p t A$ in strain LPU88 (but not in strain 2011), and (ii) whether or not such components operate in association with the Dtr or the Mpf conjugative regions. Interestingly, syntenic regions containing $r p t A$ homologs could also be identified in plasmids from the strains $S$. meliloti SM11, AK83, and GR4; S. medicae WSM419; S. fredii HH103; and Agrobacterium vitis S4; thus suggesting a more extensive requirement of $r p t A$ for plasmid mobilization (72). Analysis of plasmids in these strains suggests that the presence of $r p t A$ is associated to the type IVB Dtr-bearing plasmids.

\section{REGULATION OF THE CONJUGATIVE TRANSFER OF MEGAPLASMIDS pSymA AND pSymB IN THE MODEL STRAIN S. meliloti 1021}

As mentioned in the previous sections, studies on conjugative transfer in rhizobia have so far identified at least two different regulatory transfer systems: (i) those associated with plasmids containing type I Dtr/Mpf and exhibiting transfer regulation through a quorum sensing that responds positively to population density via $\mathrm{N}$-acylhomoserine lactones $(\underline{73}, \underline{74}, \underline{75}, \underline{76})$, and (ii) systems present in plasmids bearing type II Dtr/Mpf characterized by a so-called RctA-repressed conjugative transfer that occurs only under conditions disturbing the activity of the transcriptional repressor RctA. The rctA gene is usually associated with a virB-type T4SS (type IV secretion system); and, on the basis of this feature, several symbiotic plasmids of R. etli, S. fredii, and $S$. meliloti would belong to this category, although experimental data have been obtained so far only for the 372-kb symbiotic plasmid of R. etli CFN42, a symbiont of Phaseolus vulgaris (the common bean), and the

\footnotetext{
FIGURE 2 Phylogenetic (neighbor-joining) tree showing the relationships between different relaxases of the $\mathrm{MOB}_{\mathrm{P} / \mathrm{Q}}$ cluster as inferred from their complete protein sequences. The bootstrap-consensus tree inferred from 1,000 replicates is taken to represent the evolutionary history of the proteins (corresponding to the indicated taxa) analyzed (80). The percentage of replicate trees in which the associated taxa clustered together in the bootstrap test (1,000 replicates) is shown next to the branches. The tree is drawn to scale, with the branch lengths in proportion to the evolutionary distances used to construct the phylogenetic tree. Those distances were computed by means of the Poissoncorrection method (81) with the units being the number of amino acid substitutions per site. The protein sequences used were obtained from GenBank under the accession numbers indicated in parentheses before the name of each plasmid replicon. The box over the tree indicates the clades that include the close $\mathrm{MOB}_{\mathrm{PO}}, \mathrm{MOB}_{\mathrm{P} 3}$, and $\mathrm{MOB}_{\mathrm{P} 4}$ families of relaxases. The black dots denote those plasmid replicons that bear nodulation (nod) genes (Reprinted from Plasmid [67] with permission of the publisher). doi:10.1128/microbiolspec PLAS-0005-2013.f2
} 
1,354-Kb pSymA of S. meliloti 1021 (24). Thus far, no clues have been discerned concerning the conditions governing the conjugative transfer of plasmids either with type III Dtrs-which group includes mainly $R h i$ zobium leguminosarum plasmids that do not contain an $\mathrm{Mpf}$ system and therefore are not self-transmissible (66) -or with type IVB Dtrs/Mpfs, except for the cryptic plasmid pRmeGR4a of S. meliloti GR4, where conjugation efficiency was shown to be modulated by the nitrogen source (63). Care should be taken, however, when extrapolating phylogenetic data to mechanistic hypotheses. For instance, the plasmid pSmeSM11c - the pSymA homolog from S. meliloti SM11- was initially found to bear a type IVB Dtr/Mpf based on the phylogeny of the putative relaxase gene SM11_pC0230. However, this plasmid was also found to contain another putative relaxase (SM11_pC1037) with an associated coupling protein (SM11_pC1041) and a typical rctA gene (SM11_pC0937) linked to a virB-like Mpf operon. Thus, plasmid pSmeSM11c likely carries two different conjugative systems.

Among the pSyms, only the conjugative ability of pSymA (type II Dtr/Mpf) of S. meliloti 1021 has been established; whereas, as stated above, the pSymB behaves like a typical mobilizable element that depends on Mpf functions encoded in pSymA (25). Conjugation is regulated through the interplay of the $\mathrm{pSymA}$ transcriptional repressor RctA and the antirepressor gene $r c t B$, similar to the control that operates in the $R$. etli CFN42 pSym (24). Under conditions nonoptimal for conjugation, RctA maintains the Dtr and Mpf genes silenced. When conditions are favorable for plasmid conjugation, a transcriptional activation of $r c t B$ occurs and RctA becomes inhibited, thereby allowing expression of the conjugative genes. The mechanism of action of $r c t B$ is unknown and its predicted product shows no significant homology to proteins in existing databases. In strain $S$. meliloti 1021 , activation of $r c t B$ has recently been found to require the prior inhibition of another transcriptional repressor, RctR, that belongs to the GntR family of one-component regulatory systems (59). The DNA-binding activity of this family of regulators is usually modulated through interaction with a chemical ligand (77). According to available data, RctR does not directly repress $r c t B$ transcription. Instead, RctR represses the transcription of two adjacent gene operons, Sma0950/53, encoding an ABC transporter, and Sma0956/61, which encodes for several enzymes plus the transcriptional activator $r c t C$ (Sma0961; Fig. 3). The $\mathrm{RctC}$ gene product, in turn, appears to activate transcription of the antirepressor $r c t B(\underline{59})$. It has been suggested that an as-yet-unknown compound would be imported by the $r c t R$-adjacent $\mathrm{ABC}$ transporter, and that that signal compound or a metabolic derivative would bind the repressor RctR. That binding of the compound to RctR would thus relieve repression of the adjacent operons Sma0950/53 and Sma0956/61, including the activator gene $r c t C$. Once produced, the $\mathrm{RctC}$ protein activates transcription at the antirepressor locus $r c t B$ and the RctA repressor becomes inhibited, thus allowing plasmid conjugation to proceed. Whether or not phosphorylation of the response-regulator protein $\mathrm{RctC}$ is needed for conjugative transfer or whether the active form of RctC binds directly or not to the promoter region of $r c t B$ have not yet been investigated. The nature and origin of the signal compound(s) derepressing conjugative functions are still unknown. The RctR-RctC system is also present in other RctA plasmids-i.e., the pSym of strain S. meliloti GR4, the symbiotic plasmids of different $S$. fredii strains (e.g., NGR234, HH103, and USDA257), in addition to the nontumorigenic plasmid pAtC58 of A. tumefaciens. This system, however, is not conserved in other RctA plasmids such as the pSym of $R$. etli CFN42 or several others of $S$. meliloti. Therefore, in these other plasmids, $r c t B$ activation must proceed through a different regulatory cascade.

Although no experimental evidence has been found that quorum-sensing mechanisms might be controlling the conjugation of RctA-containing plasmids, some indications exist of a putative relationship between the $r c t A / B$ system and population density. First, all the pSymA conjugative-transfer genes of $S$. meliloti strain 8530 (an ExpR ${ }^{+}$derivative of 1021) appear overexpressed in a mucR mutant (78). MucR has accordingly arisen as a global regulator involved in the repression at low population densities of a number of energy-consuming processes, at least some of which are also activated by quorum sensing through the ExpR/SinI system (78). In addition, the pSyms of several S. meliloti strains (e.g., SM11, AK83, BL225C, and Rm41)-those also likely harboring RctA-type plasmids but not containing the above described RctR-RctC pathway to activate $r c t B$-instead possess $l u x I$-like and/or $l u x R$ like genes adjacent to a putative $r c t B$ (J. Sanjuán, unpublished observations). The conservation of putative quorum-sensing-like genes in the vicinity of the $r c t B$ locus suggests a relationship between $r c t B$ activation and population density that certainly deserves to be investigated. What seems clear is that the primary signal(s) that drive the conjugation of the RctA-containing plasmids may be different for several of these. This 


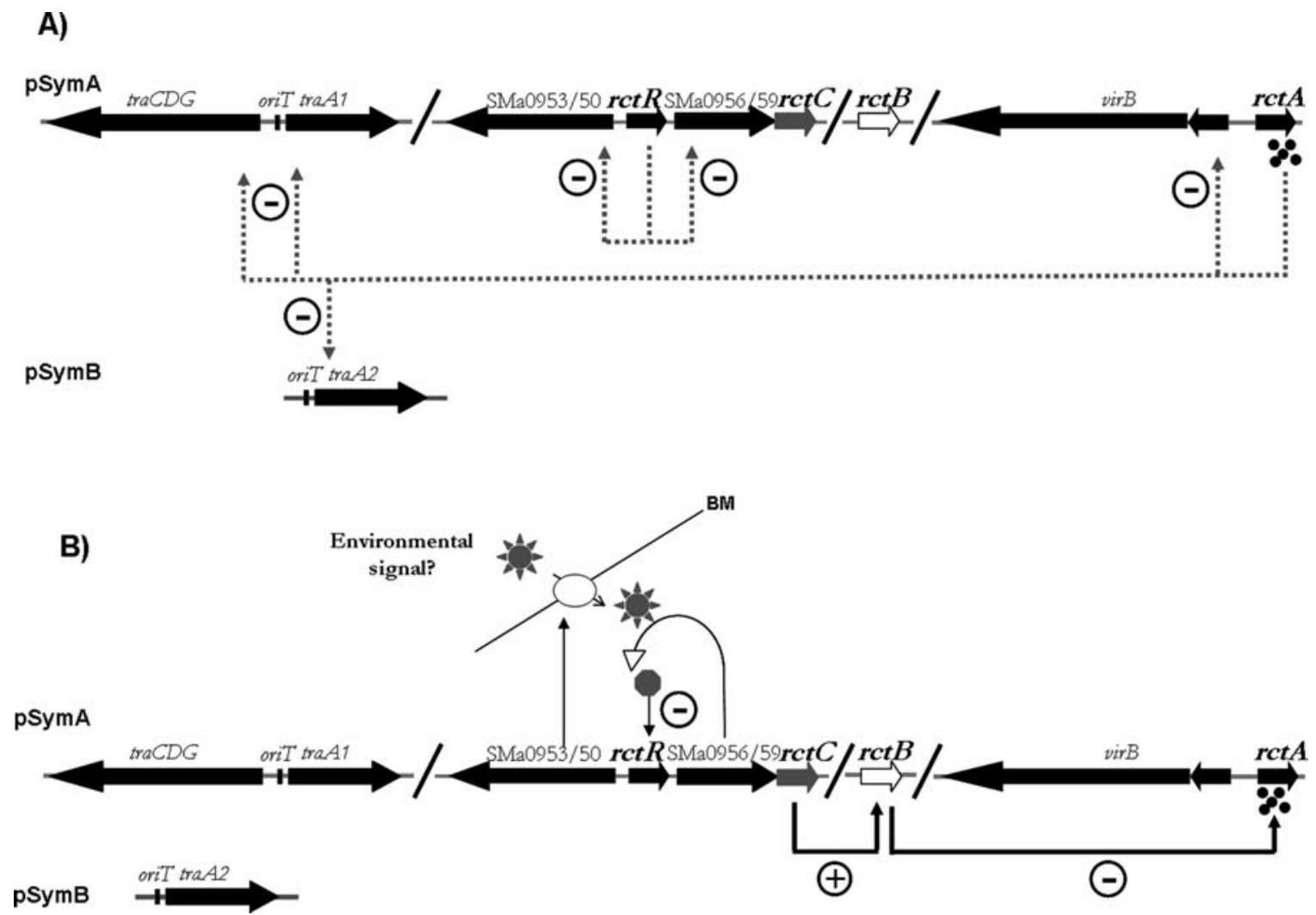

FIGURE 3 Proposed model for the regulation of conjugative transfer of the S. meliloti symbiotic plasmids. (A) Under nonfavorable conditions (i.e., standard laboratory media), RctR silences the Sma0953/50- and SMa0956-58-59-rctC operons, so that $r c t B$ remains inactive and RctA represses conjugative transfer in both pSyms. (B) Conjugative transfer is achieved after an unknown compound-putatively imported by the $A B C$ transporter encoded by SMa0953-50 and modified by the SMa0956-58-59 gene products-binds RctR, thereby relieving transcription repression of $r c t C$. The resulting $r c t C$ transcription ends up with the activation of $r c t B$ to counteract RctA, the conjugative-transfer operons become expressed, and plasmid conjugation is able to proceed. (+) activation; (-) repression or inhibition; BM, bacterial membrane (Reprinted from Environ Microbiol [59] with permission of the publisher). doi: 10.1128/microbiolspec.PLAS-0005-2013.f3

qualification together with a possible connection with population-density cues suggests a complexity of conditions that likely need to be satisfied for activating the conjugative transfer of these plasmids.

\section{DIVING INTO THE NON-pSym PLASMID COMPARTMENT AT AN OMIC SCALE}

In a recent communication, plasmids of up to 200 to $300 \mathrm{~kb}$ from $18 \mathrm{~S}$. meliloti strains (18) were separated from the chromosomal DNA and ca. 1.5 Mb of nonredundant sequence could be collected by means of highthroughput technologies (López et al., presented at the International Society for Plasmid Biology Conference, Santander, Spain, 2012). The work constituted the first deep-sequencing approach aimed at the massive characterization of the non-pSym information in S. meliloti. The sequence analysis of plasmid replication and mobilization elements evidenced an intricate phylogenetic distribution when compared with homologous elements from other rhizobia, thus pointing to the existence of an interspecific horizontal plasmid exchange. Interestingly, the mobilome sequence revealed a significant diversity of relaxases, in addition to the $\mathrm{MOB}_{\mathrm{P} 0}$ subfamily described previously. As to the kind of open reading frames present in the non-pSym mobilome, the proportion of COG classes showed clear differences compared with those from the chromosome, $\mathrm{pSymA}$, and $\mathrm{pSymB}$ (A. Lagares and M. Pistorio, unpublished results); indicating clear functional differentiations among all four 
of these genetic compartments. The experimental approach used proved to be feasible, with deep penetration down to the scale of single-gene analysis. The thousands of genes collected other than those associated with the basic plasmid biology (replication/maintenance/transfer) represent, certainly, the most valuable portion of the new information available.

\section{CONCLUDING REMARKS}

The split configuration of the bacterial genome qualitatively into chromosome(s) along with plasmids as intercellular mobile genetic vectors is a cogent physical indication of an efficient and extensive ancestral mechanism of communal evolution and adaptation in prokaryotes. The separation of the essential housekeeping functions resident in the chromosomes from those associated with adaptive responses encoded in plasmid enables bacteria to access and manage, at the population level, an enormous and diverse amount of (horizontally) shareable and accessible information (i.e., the mobilome) that could not otherwise be handled by single bacterial cells, with the final result being that the community as a whole evolves (79). Rhizobia, in general, and $S$. meliloti, in particular, are soil bacteria where their plasmid replicons are known to provide the genetic basis both for speciation events (i.e., HGT of symbiotic traits) and for environmental adaptation. The available information demonstrates that the $S$. meliloti plasmid compartment includes replicons with a wide range of sizes, diverse gene contents, and different degrees of conservation. Among the different strains, diverse kinds of conjugative genes along with their associated regulatory mechanisms were described, with clear evidences of coevolution. A solid understanding of the conjugative mechanisms that mediate both transient and longterm genomic changes in $S$. meliloti will be necessary to help unravel fundamental questions such as how these rhizobia were born as plant symbionts, how their symbioses and specificities evolved, how active the exchange of genetic information with other rhizobia is, and mdash;related to this last question-how ubiquitous the $S$. meliloti conjugative systems are in other plantassociated sympatric rhizospheric-soil bacteria. Upcoming research is expected to focus with much more intensity on the analysis of the (still mostly hidden) genes and functions encoded in the S. meliloti non-pSymplasmid pool. Information of that sort is expected to further our understanding of the diversity of processes that contribute to the symbiotic and free-living lifestyles of these rhizobia.

\section{ACKNOWLEDGMENTS}

Drs Lagares and Pistorio are funded by CONICET (Consejo Nacional de Investigaciones Científicas y Técnicas - Argentina), MinCyT (Ministerio de Ciencia Tecnolología e Innovación Productiva - MinCyT-Argentina; PICT 2005-31937, PICT 2008-0736, PICT 2012-0102; PICT 2012-0518; PICT 20121719), and the European Commission (FP7, Metaexplore, KBBE222625). Dr Sanjuán is funded by CSIC (Consejo Superior de Investigaciones Científicas, Spain), Consejería de Economía, Innovación, Ciencia y Empleo de la Junta de Andalucía, Spain (grant AGR-258), and Plan Andaluz de Investigación, Spain.

Conflicts of interest: We declare that we have no conflicts.

\section{REFERENCES}

1. Peoples MB, Craswell ET. 1992. Biological nitrogen fixation: investments, expectations and actual contributions to agriculture. Plant Soil 141:13-39.

2. Peoples MB, Herridge DF. 1990. Nitrogen fixation by legumes in tropical and sub-tropical agriculture. Adv Agron 44:155-224.

3. García de los Santos A, Brom S, Romero D. 1996. Rhizobium plasmids in bacteria-legume interactions. World J Microbiol Biotechnol 12:119-125. 4. Mercado-Blanco J, Toro N. 1996. Plasmids in Rhizobia: the role of nonsymbiotic plasmids. Mol Plant Microbe Interact 9:535-545.

5. Banfalvi Z, Kondorosi E, Kondorosi A. 1985. Rhizobium meliloti carries two megaplasmids. Plasmid 13:129-138.

6. Hynes MF, Simon R, Müller P, Niehaus K, Labes M, Pühler A. 1986. The two megaplasmids of Rhizobium meliloti are involved in the effective nodulation of alfalfa. Mol Gen Genet 202:356-362.

7. Banfalvi Z, Sakanyan V, Koncz C, Kiss A, Dusha I, Kondorosi A. 1981. Location of nodulation and nitrogen fixation genes on a high molecular weight plasmid of Rhizobium meliloti. Mol Gen Genet 184:318-325.

8. Smets BF, Barkay T. 2005. Horizontal gene transfer: perspectives at a crossroads of scientific disciplines. Nat Rev Microbiol 3:675-678.

9. Pistorio M, Del Papa MF, Balague LJ, Lagares A. 2003. Identification of a transmissible plasmid from an Argentine Sinorhizobium meliloti strain which can be mobilised by conjugative helper functions of the European strain S. meliloti GR4. FEMS Microbiol Lett 225:15-21.

10. Roumiantseva ML, Andronov EE, Sharypova LA, DammannKalinowski T, Keller M, Young JP, Simarov BV. 2002. Diversity of Sinorhizobium meliloti from the Central Asian alfalfa gene center. Appl Environ Microbiol 68:4694-4697.

11. Stiens M, Schneiker S, Keller M, Kuhn S, Pühler A, Schlüter A. 2006. Sequence analysis of the 144-kilobase accessory plasmid pSmeSM11a, isolated from a dominant Sinorhizobium meliloti strain identified during a long-term field release experiment. Appl Environ Microbiol 72:36623672 .

12. Kosier B, Pühler A, Simon R. 1993. Monitoring the diversity of Rhizobium meliloti field and microcosm isolates with a novel rapid genotyping method using insertion elements. Mol Ecol 2:35-36.

13. Pistorio M, Giusti MA, Del Papa MF, Draghi WO, Lozano MJ, Tejerizo GT, Lagares A. 2008. Conjugal properties of the Sinorhizobium meliloti plasmid mobilome. FEMS Microbiol Ecol 65:372-382.

14. Mercado-Blanco J, Olivares J. 1993. Stability and transmissibility of the cryptic plasmids of Rhizobium meliloti GR4. Their possible use in the construction of cloning vectors of rhizobia. Arch Microbiol 160:477-485.

15. Barnett MJ, Fisher RF, Jones T, Komp C, Abola AP, Barloy-Hubler F, Bowser L, Capela D, Galibert F, Gouzy J, Gurjal M, Hong A, Huizar L, Hyman RW, Kahn D, Kahn ML, Kalman S, Keating DH, Palm C, Peck MC, Surzycki R, Wells DH, Yeh KC, Davis RW, Federspiel NA, Long SR. 2001. Nucleotide sequence and predicted functions of the entire Sinorhizobium meliloti pSymA megaplasmid. Proc Natl Acad Sci USA 98:9883-9888. 
16. Finan TM, Weidner S, Wong K, Buhrmester J, Chain P, Vorholter FJ, Hernandez-Lucas I, Becker A, Cowie A, Gouzy J, Golding B, Puhler A. 2001. The complete sequence of the 1,683-kb pSymB megaplasmid from the $\mathrm{N}_{2}$-fixing endosymbiont Sinorhizobium meliloti. Proc Natl Acad Sci USA 98:9889-9894.

17. Giuntini E, Mengoni A, De Filippo C, Cavalieri D, Aubin-Horth N, Landry CR, Becker A, Bazzicalupo M. 2005. Large-scale genetic variation of the symbiosis-required megaplasmid pSymA revealed by comparative genomic analysis of Sinorhizobium meliloti natural strains. BMC Genomics 6:158. doi:10.1186/1471-2164-6-158.

18. Oresnik IJ, Liu SL, Yost CK, Hynes MF. 2000. Megaplasmid pRme2011a of Sinorhizobium meliloti is not required for viability. J Bacteriol 182:3582-3586.

19. Slater SC, Goldman BS, Goodner B, Setubal JC, Farrand SK, Nester EW, Burr TJ, Banta L, Dickerman AW, Paulsen I, Otten L, Suen G, Welch R, Almeida NF, Arnold F, Burton OT, Du Z, Ewing A, Godsy E, Heisel S, Houmiel KL, Jhaveri J, Lu J, Miller NM, Norton S, Chen Q, Phoolcharoen W, Ohlin V, Ondrusek D, Pride N, Stricklin SL, Sun J, Wheeler C, Wilson L, Zhu H, Wood DW. 2009. Genome sequences of three agrobacterium biovars help elucidate the evolution of multichromosome genomes in bacteria. J Bacteriol 191:2501-2511.

20. Galardini M, Pini F, Bazzicalupo M, Biondi EG, Mengoni A. 2013. Replicon-dependent bacterial genome evolution: the case of Sinorhizobium meliloti. Genome Biol Evol 5:542-558.

21. Harrison PW, Lower RP, Kim NK, Young JP. 2010. Introducing the bacterial 'chromid': not a chromosome, not a plasmid. Trends Microbiol 18:141-148.

22. Galardini M, Mengoni A, Brilli M, Pini F, Fioravanti A, Lucas S, Lapidus A, Cheng JF, Goodwin L, Pitluck S, Land M, Hauser L, Woyke T, Mikhailova N, Ivanova N, Daligault H, Bruce D, Detter C, Tapia R, Han C, Teshima H, Mocali S, Bazzicalupo M, Biondi EG. 2011. Exploring the symbiotic pangenome of the nitrogen-fixing bacterium Sinorhizobium meliloti. BMC Genomics 12:235. doi:10.1186/1471-2164-12-235.

23. Schneiker-Bekel S, Wibberg D, Bekel T, Blom J, Linke B, Neuweger H, Stiens M, Vorholter FJ, Weidner S, Goesmann A, Puhler A, Schluter A. 2011. The complete genome sequence of the dominant Sinorbizobium meliloti field isolate SM11 extends the S. meliloti pan-genome. J Biotechnol 155:20-33.

24. Perez-Mendoza D, Sepulveda E, Pando V, Munoz S, Nogales J, Olivares J, Soto MJ, Herrera-Cervera JA, Romero D, Brom S, Sanjuan J. 2005. Identification of the $r c t A$ gene, which is required for repression of conjugative transfer of rhizobial symbiotic megaplasmids. J Bacteriol 187:7341-7350.

25. Blanca-Ordóñez H, Oliva-García JJ, Pérez-Mendoza D, Soto MJ, Olivares J, Sanjuán J, Nogales J. 2010. pSymA-dependent mobilization of the Sinorhizobium meliloti pSymB megaplasmid. J Bacteriol 192:63096312.

26. Barran LR, Ritchot N, Bromfield ES. 2001. Sinorhizobium meliloti plasmid $\mathrm{pRm} 1132 \mathrm{f}$ replicates by a rolling-circle mechanism. J Bacteriol 183:2704-2708.

27. Hahn M, Hennecke H. 1987. Mapping of a Bradyrhizobium japonicum DNA region carrying genes for symbiosis and an asymmetric accumulation of reiterated sequences. Appl Environ Microbiol 53:22472252.

28. Kaluza K, Hahn M, Hennecke H. 1985. Repeated sequences similar to insertion elements clustered around the nif region of the Rhizobium japonicum genome. J Bacteriol 162:535-542.

29. Bromfield ES, Lewis DM, Barran LR. 1985. Cryptic plasmid and rifampin resistance in Rhizobium meliloti influencing nodulation competitiveness. J Bacteriol 164:410-413.

30. García-Rodriguez FM, Toro N. 2000. Sinorbizobium meliloti nfe (nodulation formation efficiency) genes exhibit temporal and spatial expression patterns similar to those of genes involved in symbiotic nitrogen fixation. Mol Plant Microbe Interact 13:583-591.
31. Soto MJ, Zorzano A, Garcia-Rodriguez FM, Mercado-Blanco J, Lopez-Lara IM, Olivares J, Toro N. 1994. Identification of a novel Rhizobium meliloti nodulation efficiency nfe gene homolog of Agrobacterium ornithine cyclodeaminase. Mol Plant Microbe Interact 7:703707.

32. Soto MJ, Zorzano A, Mercado-Blanco J, Lepek V, Olivares J, Toro N. 1993. Nucleotide sequence and characterization of Rhizobium meliloti nodulation competitiveness genes nfe. J Mol Biol 229:570-576.

33. Velázquez E, Mateos PF, Pedrero P, Dazzo FB, Martinez-Molina E. 1995. Attenuation of symbiotic effectiveness by Rhizobium meliloti SAF22 related to the presence of a cryptic plasmid. Appl Environ Microbiol 61:2033-2036.

34. Eckhardt T. 1978. A rapid method for the identification of plasmid desoxyribonucleic acid in bacteria. Plasmid 1:584-588.

35. Wheatcroft R, McRae DG, Miller RW. 1990. Changes in the Rhizobium meliloti genome and the ability to detect supercoiled plasmids during bacteroid development. Mol Plant Microbe Interact 3:9-17.

36. Plazinski J, Cen YH, Rolfe BG. 1985. General method for the identification of plasmid species in fast-growing soil microorganisms. Appl Environ Microbiol 49:1001-1003.

37. Velázquez E, Mateos PF, Velasco N, Santos F, Burgos PA, Villadas P, Toro N, Martínez-Molina E. 1999. Symbiotic characteristics and selection of autochthonous strains of Sinorhizobium meliloti populations in different soils. Soil Biol Biochem 31:1039-1047.

38. Coutinho HLC, Oliveira VM, Lovato A, Maia AHN, Manfio GP. 1999. Evaluation of the diversity of rhizobia in Brazilian agricultural soils cultivated with soybeans. Appl Soil Ecol 13:159-167.

39. Cevallos MA, Cervantes-Rivera R, Gutierrez-Rios RM. 2008. The repABC plasmid family. Plasmid 60:19-37.

40. Pinto UM, Pappas KM, Winans SC. 2012. The ABCs of plasmid replication and segregation. Nat Rev Microbiol 10:755-765.

41. Ramírez-Romero MA, Soberón N, Pérez-Oseguera A, Téllez-Sosa J, Cevallos MA. 2000. Structural elements required for replication and incompatibility of the Rhizobium etli symbiotic plasmid. J Bacteriol 182: 3117-3124.

42. Salje J. 2010. Plasmid segregation: how to survive as an extra piece of DNA. Crit Rev Biochem Mol Biol 45:296-317.

43. Hwang LC, Vecchiarelli AG, Han YW, Mizuuchi M, Harada Y, Funnell BE, Mizuuchi K. 2013. ParA-mediated plasmid partition driven by protein pattern self-organization. EMBO J 32:1238-1249.

44. Cevallos MA, Porta H, Izquierdo J, Tun-Garrido C, Garcia-de-losSantos A, Davila G, Brom S. 2002. Rhizobium etli CFN42 contains at least three plasmids of the $\operatorname{rep} A B C$ family: a structural and evolutionary analysis. Plasmid 48:104-116.

45. Venkova-Canova T, Soberón NE, Ramírez-Romero MA, Cevallos MA. 2004. Two discrete elements are required for the replication of a repABC plasmid: an antisense RNA and a stem-loop structure. Mol Microbiol 54:1431-1444.

46. Chai Y, Winans SC. 2005. A small antisense RNA downregulates expression of an essential replicase protein of an Agrobacterium tumefaciens Ti plasmid. Mol Microbiol 56:1574-1585.

47. MacLellan SR, Smallbone LA, Sibley CD, Finan TM. 2005. The expression of a novel antisense gene mediates incompatibility within the large repABC family of alpha-proteobacterial plasmids. Mol Microbiol 55:611-623.

48. Mercado-Blanco J, Olivares J. 1994. The large nonsymbiotic plasmid pRmeGR4a of Rhizobium meliloti GR4 encodes a protein involved in replication that has homology with the RepC protein of Agrobacterium plasmids. Plasmid 32:75-79.

49. Izquierdo J, Venkova-Canova T, Ramirez-Romero MA, Tellez-Sosa J, Hernandez-Lucas I, Sanjuan J, Cevallos MA. 2005. An antisense RNA plays a central role in the replication control of a repC plasmid. Plasmid 54:259-277. 
50. Watson RJ, Heys R. 2006. Replication regions of Sinorhizobium meliloti plasmids. Plasmid 55:87-98.

51. Castellanos M, Romero D. 2009. The extent of migration of the Holliday junction is a crucial factor for gene conversion in Rhizobium etli. $J$ Bacteriol 191:4987-4995.

52. Hernandez-Salmeron JE, Santoyo G. 2011. Phylogenetic analysis reveals gene conversions in multigene families of rhizobia. Genet Mol Res 12:1383-1392.

53. Tian CF, Young JP, Wang ET, Tamimi SM, Chen WX. 2010. Population mixing of Rhizobium leguminosarum bv. viciae nodulating Vicia faba: the role of recombination and lateral gene transfer. FEMS Microbiol Ecol 73:563-576.

54. Torres Tejerizo G, Del Papa MF, Giusti MA, Draghi W, Lozano M, Lagares A, Pistorio M. 2010. Characterization of extrachromosomal replicons present in the extended host range Rhizobium sp. LPU83. Plasmid 64:177-185.

55. van Berkum P, Terefework Z, Paulin L, Suomalainen S, Lindstrom K, Eardly BD. 2003. Discordant phylogenies within the $r r n$ loci of rhizobia. $J$ Bacteriol 185:2988-2998.

56. Cervantes L, Bustos P, Girard L, Santamaría RI, Dávila G, Vinuesa P, Romero D, Brom S. 2011. The conjugative plasmid of a bean-nodulating Sinorbizobium fredii strain is assembled from sequences of two Rhizobium plasmids and the chromosome of a Sinorhizobium strain. BMC Microbiol 11:149. doi:10.1186/1471-2180-11-149.

57. Mazur A, Majewska B, Stasiak G, Wielbo J, Skorupska A. 2011. repABC-based replication systems of Rhizobium leguminosarum bv. trifolii TA1 plasmids: incompatibility and evolutionary analyses. Plasmid 66:53-66. 58. Suominen L, Roos C, Lortet G, Paulin L, Lindstrom K. 2001. Identification and structure of the Rhizobium galegae common nodulation genes: evidence for horizontal gene transfer. Mol Biol Evol 18:907-916. 59. Nogales J, Blanca-Ordonez H, Olivares J, Sanjuan J. 2013. Conjugal transfer of the Sinorhizobium meliloti 1021 symbiotic plasmid is governed through the concerted action of one- and two-component signal transduction regulators. Environ Microbiol 15:811-821.

60. Bailly X, Olivieri I, Brunel B, Cleyet-Marel JC, Béna G. 2007. Horizontal gene transfer and homologous recombination drive the evolution of the nitrogen-fixing symbionts of Medicago species. J Bacteriol 189: 5223-5236.

61. Shannon CE, Weaver W. 1949. Evolution and measurement of species diversity. Taxon 21:213-251.

62. Simpson EH. 1949. Measurement of diversity. Nature 163:688.

63. Herrera-Cervera JA, Olivares J, Sanjuan J. 1996. Ammonia inhibition of plasmid pRmeGR4a conjugal transfer between Rhizobium meliloti strains. Appl Environ Microbiol 62:1145-1150.

64. Giusti MA, Lozano MJ, Torres Tejerizo GA, Martini MC, Salas ME, López JL, Draghi WO, Del Papa MF, Pistorio M, Lagares A. 2013. Conjugal transfer of a Sinorhizobium meliloti cryptic plasmid evaluated during a field release and in soil microcosms. Eur J Soil Biol 55:9-12.

65. Kinkle BK, Schmidt EL. 1991. Transfer of the pea symbiotic plasmid pJB5JI in nonsterile soil. Appl Environ Microbiol 57:3264-3269.

66. Ding H, Hynes MF. 2009. Plasmid transfer systems in the rhizobia. Can J Microbiol 55:917-927.
67. Giusti MA, Pistorio M, Lozano MJ, Torres Tejerizo GA, Salas ME, Martini MC, López JL, Draghi WO, Del Papa MF, Pérez-Mendoza D, Sanjuán J, Lagares A. 2012. Genetic and functional characterization of a yet-unclassified rhizobial Dtr (DNA-transfer-and-replication) region from the ubiquitous plasmid conjugal system present in Sinorhizobium meliloti, Sinorhizobium medicae, and in other Gram-negative bacteria. Plasmid 67:199-210.

68. Ding H, Yip CB, Hynes MF. 2013. Genetic characterization of a novel rhizobial plasmid conjugation system in Rhizobium leguminosarum bv. viciae strain VF39SM. J Bacteriol 195:328-339.

69. Herrera-Cervera JA, Sanjuan-Pinilla JM, Olivares J, Sanjuan J. 1998. Cloning and identification of conjugative transfer origins in the Rhizobium meliloti genome. J Bacteriol 180:4583-4590.

70. Garcillan-Barcia MP, Francia MV, de la Cruz F. 2009. The diversity of conjugative relaxases and its application in plasmid classification. FEMS Microbiol Rev 33:657-687.

71. Holmes B, Poppoff M, Kiredjian M, Kersters K. 1988. Ochrobactrum anthropi gen. nov., sp. nov. from human clinical specimens and previously known as group Vd. Int J Syst Evol Bacteriol 38:406-416.

72. Pistorio M, Torres Tejerizo GA, Del Papa MF, de Los Angeles Giusti M, Lozano M, Lagares A. 2013. $r p t A$, a novel gene from Ensifer (Sinorhizobium) meliloti involved in conjugal transfer. FEMS Microbiol Lett 345:22-30.

73. He X, Chang W, Pierce DL, Seib LO, Wagner J, Fuqua C. 2003. Quorum sensing in Rhizobium sp. strain NGR234 regulates conjugal transfer (tra) gene expression and influences growth rate. J Bacteriol 185:809-822.

74. Danino VE, Wilkinson A, Edwards A, Downie JA. 2003. Recipientinduced transfer of the symbiotic plasmid pRL1JI in Rhizobium leguminosarum bv. viciae is regulated by a quorum-sensing relay. Mol Microbiol 50:511-525.

75. Wilkinson A, Danino V, Wisniewski-Dye F, Lithgow JK, Downie JA. 2002. N-acyl-homoserine lactone inhibition of rhizobial growth is mediated by two quorum-sensing genes that regulate plasmid transfer. $J$ Bacteriol 184:4510-4519.

76. Tun-Garrido C, Bustos P, Gonzalez V, Brom S. 2003. Conjugative transfer of $\mathrm{p} 42$ a from Rhizobium etli CFN42, which is required for mobilization of the symbiotic plasmid, is regulated by quorum sensing. J Bacteriol 185:1681-1692.

77. Hoskisson PA, Rigali S. 2009. Chapter 1: variation in form and function: the helix-turn-helix regulators of the GntR superfamily. $A d v$ Appl Microbiol 69:1-22.

78. Mueller K, Gonzalez JE. 2011. Complex regulation of symbiotic functions is coordinated by MucR and quorum sensing in Sinorbizobium meliloti. J Bacteriol 193:485-496.

79. Woese CR. 2002. On the evolution of cells. Proc Natl Acad Sci USA 99:8742-8747.

80. Felsenstein J. 1985. Confidence limits on phylogenies: an approach using the bootstrap. Evolution 39:783-791.

81. Zuckerkandl E, Pauling L. 1965. Evolutionary divergence and convergence in proteins, p 97-166. In Bryson V, Vogel HJ (ed), Evolving Genes and Proteins. Academic Press, New York, NY. 


\section{Author Queries}

Q1: For Lopez et al., please provide all author names and initials, and the range of dates for the meeting in Santander. 